\title{
INSECURE PROPERTY RIGHTS AND GROWTH: THE ROLES OF APPROPRIATION COSTS, WEALTH EFFECTS, AND HETEROGENEITY
}

\author{
NGO VAN LONG \\ GERHARD SORGER
}

\begin{abstract}
CESIFO WORKING PAPER NO. 1253
CATEGORY 5: FisCAL POLICY, MACROECONOMICS AND GROWTH

August 2004
\end{abstract}

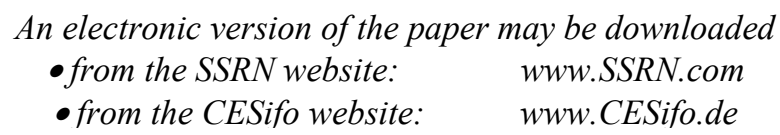




\title{
INSECURE PROPERTY RIGHTS AND GROWTH: THE ROLES OF APPROPRIATION COSTS, WEALTH EFFECTS, AND HETEROGENEITY
}

\begin{abstract}
We extend the model of insecure property rights by Tornell and Velasco (1992) and Tornell and Lane (1999) by adding three features: (i) extracting the common property asset involves a private appropriation cost, (ii) agents derive utility from wealth as well as from consumption, and (iii) agents can be heterogeneous. We show that both an increase in the appropriation cost and, when appropriation costs vary across agents, an increase in the degree of heterogeneity of these costs reduce the growth rate of the public capital stock. We also show that, in the interior equilibrium, the private asset can have either a lower or a higher money rate of return than the common property asset.
\end{abstract}

JEL Code: C73, O40.

Keywords: corruption, property rights, growth, appropriation cost.

Ngo Van Long

Dept of Economics

National Singapore University

Singapore 117570

ngo.long@mcgill.ca
Gerhard Sorger

Department of Economics

University of Vienna

Hohenstaufengasse 9

A-1010 Vienna

Austria 


\section{Introduction}

Recently, there has been increased interest in studying economic growth in the absence of secure property rights and the presence of multiple organized and, hence, powerful groups in society; see, e.g., Tornell [9], Tornell and Lane [5, 10], Tornell and Velasco [11], or Lindner and Strulik [6]. When legal or political institutions are weak, powerful groups can influence the fiscal process and thereby in effect redistribute the economywide capital stock among themselves. The development of the economy is therefore more appropriately described by a dynamic resource allocation game than by a traditional neoclassical growth model with perfect competition. Pursuing this line of thought, the above mentioned papers study one-sector growth models with finitely many agents (the power groups) who share access to the economy's capital stock. Because the capital stock in these models is a common property asset, the familiar tragedy of the commons becomes relevant: the powerful groups do not internalize the negative effects which their appropriation efforts have on the production capacity of the entire economy and, hence, economic growth is inefficiently low.

Tornell and Velasco [11] and Tornell and Lane [10] add another interesting feature to this general setup: they assume that the players can extract resources from the public and insecure capital stock and convert them into private and secure asset holdings. The private asset stocks could be interpreted, for example, as bank accounts in foreign countries in which property rights are secure. In the models studied in Tornell and Velasco [11] and Tornell and Lane [10], the extraction from the common property asset stock is costless and the authors claim that "including appropriation or adjustment costs would add nothing to the insights provided by the model" [10, p. 26]. ${ }^{1}$ We believe, however, that an explicit consideration of costs of appropriation is important. A model that takes appropriation costs into account is not only more realistic (after all, money laundering and lobbying involve the use of real resources) but, as our analysis shows, it also yields new insights and modifies a few key results from Tornell and Velasco [11] and Tornell and Lane [10] in non-trivial ways. As for the new results, we can show for example that both an increase in the appropriation cost and, when appropriation costs vary across agents, an increase in the degree of heterogeneity of these costs reduce the growth rate of the public capital stock. Thus, we have the striking result that high costs of money laundering are detrimental to economic growth.

We also add another feature to the analysis, namely that the agents derive utility not only from consumption but also from their wealth. Wealth is a vehicle for achieving social status, and people do care about social status. Cole et al. [1] argue forcefully that status seeking is a strong motive for economic agents, especially if goods are not allocated through well-functioning markets. ${ }^{2}$ Including wealth as a ranking device in the utility functions of the power groups

\footnotetext{
${ }^{1}$ Their main results are as follows. First, the existence of powerful groups reduces the growth rate. Second, an increase in the number of groups leads to better economic performance. And, third, an increase in the raw rate of return to the common property asset reduces its growth rate (the voracity effect).

${ }^{2}$ Cole et al. [1, p. 1092]) quote from The Theory of Moral Sentiments by Adam Smith: "To what purpose is all the toil and bustle of the world? ... It is our vanity that urges us on. ... It is not wealth that men desire, but the consideration and good opinion that wait upon riches." Cole et al. [1] also quote from Material Girl by Madonna: "The boy with the cold hard cash is always Mister Right because we are living in the material world
} 
allows us to study how the elasticity of substitution between wealth and consumption affects the equilibrium outcome. In particular, we are able to derive a simple condition that ensures that higher substitutability leads to a higher intensity of extraction from the common property asset and therefore to a smaller growth rate of the public capital stock. The combination of the wealth effect and positive appropriation costs has also another interesting consequence. Whereas in the model of Tornell and Velasco [11] and Tornell and Lane [10] the equilibrium money returns on both types of assets must coincide, this is no longer true in our model with wealth effects and positive costs of transferring resources from the public to the private asset. As a matter of fact, depending on the parameter values, the private asset can have either a lower or a higher money rate of return than the public one. ${ }^{3}$

Considering the domestic capital stock as a common property asset makes the model formally similar to one in which agents have common access to a natural (renewable or non-renewable) resource. There is a large literature on these models and the paper that is closest to ours in this respect is the one by Sorger [8]. Resource extraction models that allow for private storage of the extracted resource have been studied, for example, by Sinn [7], Kremer and Morcom [4], Gaudet et al. [3], or Dutta and Rowat [2]. Whereas Kremer and Morcom [4] and Gaudet et al. [3] study competitive resource markets, Sinn [7] and Dutta and Rowat [2] deal with oligopolistic markets. The paper by Dutta and Rowat [2] uses a setup that is very close to ours except that it does not include wealth-dependent utility. Their focus, however, is quite different from ours since they are mostly interested in whether or not extinction can occur as an equilibrium outcome. In our model, the marginal utility of wealth becomes infinitely large as wealth approaches 0 , which implies that agents will never run down their wealth in finite time, i.e., extinction is not possible in equilibrium.

The paper is organized as follows. In section 2 we formulate the model and state all assumptions. Section 3 characterizes the interior equilibrium under the assumption of homogeneous players. We discuss how the structure of the equilibrium is affected by the presence of appropriation costs and wealth effects. Furthermore, we study how changes in one or more model parameters influence the intensity of appropriation and the net growth rate of the public capital stock. Section 4 introduces heterogeneity of the power groups. We characterize again an interior Nash equilibrium of the game and analyze how the growth rate of the economy depends on the degree of heterogeneity. Finally, in section 5 we impose an additional constraint on the intensity of extraction from the public capital stock. Such a constraint can be interpreted in terms of capital controls. We show that, in general, there exists a pessimistic equilibrium in addition to the interior equilibrium discussed in section 3. In the pessimistic equilibrium all players transfer resources from the public capital stock into their private asset holdings as quickly as possible. Finally, section 6 presents concluding remarks.

and I am a material girl."

${ }^{3}$ Of course, if one defines appropriate concepts such as 'full rates of return', then, by definition, they must be equalized in equilibrium. 


\section{Model formulation}

Time is modelled as a continuous variable. Consider a common property asset with constant rate of return $R$. We interpret this asset as the aggregate capital stock in an economy with insecure property rights. There are $n$ identical agents with access to this asset. Each agent represents a group of individuals who - because they are organized and because property rights are poorly defined or not fully enforced - can influence the allocation of the capital stock among the $n$ groups. We do not explicitly model how the groups can redistribute capital: this can happen, for example, through their influence on the fiscal process, through lobbying activities or corruption, or through forcible misappropriation. ${ }^{4}$ Adopting the simplifications made in the literature cited at the opening of the introduction, we assume that the agents can withdraw from the public asset stock but that they cannot invest into it. The withdrawal rate (or extraction rate) of agent $i$ at time $t$ from the common property asset will be denoted by $x_{i}(t)$, the asset stock itself at time $t$ is denoted by $z(t)$, and the initial value at time 0 is denoted by $z_{0}$. The aggregate capital stock evolves therefore according to the differential equation

$$
\dot{z}(t)=R z(t)-\sum_{i=1}^{n} x_{i}(t), z(0)=z_{0} .
$$

Resources that the agents extract from the aggregate capital stock can be either consumed or invested into private and secure assets. These private assets can be interpreted as safe bank accounts in foreign countries, where property rights are fully enforced. The rate of return on the private asset is constant and given by $r$. Denoting the rate of consumption of agent $i$ at time $t$ by $c_{i}(t)$, it follows that investment into the private asset holdings of agent $i$ at time $t$ is given by $x_{i}(t)-c_{i}(t)$. Let us denote the private asset stock of agent $i$ at time $t$ by $y_{i}(t)$ and its initial value at time 0 is $y_{i 0}$. It follows that

$$
\dot{y}_{i}(t)=r y_{i}(t)+x_{i}(t)-c_{i}(t), y_{i}(0)=y_{i 0}
$$

for all $i$ and all $t$. Consumption must be non-negative at all times. We also assume that the agents cannot incur debt, that is, the private asset holdings must be non-negative. The feasibility conditions can therefore be summarized by the requirement that for all $t$ and all $i$ the inequalities

$$
x_{i}(t) \geq 0, c_{i}(t) \geq 0, y_{i}(t) \geq 0, z(t) \geq 0
$$

are satisfied.

Let us define

$$
A_{i}(t)=y_{i}(t)+\gamma z(t)
$$

where $\gamma$ is a non-negative parameter. We may interpret $A_{i}(t)$ as the total wealth of agent $i$ at time $t$, where $\gamma$ measures the weight given to public asset holdings relative to private asset holdings. The weight $\gamma$ can be smaller or larger than 1 , depending on whether the agents attach more or less importance to their private asset stock. For example, it may be that $\gamma=1 / n$,

\footnotetext{
${ }^{4}$ Tornell and Lane [10] develop a detailed model of how powerful groups can redistribute capital through the fiscal process.
} 
which would indicate that each agent thinks that only a fraction of the common asset really belongs to him. Alternatively, the agents may give more weight to the public asset because the private asset holdings are illegal and cannot be used to indicate high status. Anyway, in our general formulation we allow $\gamma$ to take any non-negative value. The agents derive utility both from consumption and from wealth. We denote the instantaneous utility of agent $i$ derived at time $t$ by $U\left(c_{i}(t), A_{i}(t)\right)$. The presence of $A_{i}(t)$ as an argument of the utility function displays a wealth effect that has so far been ignored in the literature on growth under insecure property rights.

We assume that both investment into and withdrawal from the private asset holdings are costless. Extraction from the common property asset, however, is assumed to be costly. This reflects the fact that considerable resources have to be spent for lobbying or money laundering. The marginal cost of appropriation from the common property asset measured in units of utility is assumed to be constant and will be denoted by $\kappa$. Finally, we assume that the common time-preference rate of all agents is given by $\rho$. Agent $i$ therefore seeks to maximize

$$
\int_{0}^{+\infty} e^{-\rho t}\left[U\left(c_{i}(t), A_{i}(t)\right)-\kappa x_{i}(t)\right] \mathrm{d} t
$$

subject to constraints (1)-(4).

As for the parameters of the model we make the following assumption.

Assumption P: The number of players is an integer $n \geq 2$. The parameters $R, z_{0}, r, y_{i 0}, \gamma$, $\kappa$, and $\rho$ are real numbers satisfying $z_{0}>0, y_{i 0} \geq 0, \gamma \geq 0, \rho>0$, and $\rho>r$.

The instantaneous utility function $U$ is assumed to be homogeneous of degree 1 . This implies that $U(c, A)=A u(q)$, where $q=c / A$ is the ratio of consumption to wealth and where the function $u$ is defined by the equation $u(q)=U(q, 1)$. The properties of $U$, which we shall assume throughout the analysis, are summarized next.

Assumption U: The instantaneous utility function $U:[0,+\infty) \times[0, \infty) \mapsto \mathbb{R}$ is concave and homogeneous of degree 1 on its domain. The function $u:[0,+\infty) \mapsto \mathbb{R}$ defined by $u(q)=U(q, 1)$ is continuous, strictly increasing, and strictly concave on its domain and it satisfies $u(0)=0$. Furthermore, $u$ is assumed to be continuously differentiable on the interior of its domain and to satisfy the Inada-type conditions $\lim _{q \rightarrow 0} u^{\prime}(q)=+\infty$ and $\lim _{q \rightarrow+\infty} u^{\prime}(q)=0$.

Assumptions $\mathrm{P}$ and $\mathrm{U}$ will be maintained throughout the rest of the paper without further mentioning. It is well-known that $\partial U(c, A) / \partial c=u^{\prime}(c / A)$ holds for all $(c, A)$ in the interior of the domain of $U$. In other words, the marginal utility of consumption depends only on the consumption/wealth ratio $q$. Analogously, the marginal utility of wealth is given by $\partial U(c, A) / \partial A=w(c / A)$, where $w(q)=u(q)-u^{\prime}(q) q$ is a strictly positive and strictly increasing function of $q=c / A>0$.

We are interested in symmetric Markov-perfect Nash equilibria of the differential game specified above. These equilibria are defined as follows. Let $\mathbf{y}(t)=\left(y_{1}(t), y_{2}(t), \ldots, y_{n}(t)\right) \in[0,+\infty)^{n}$ be the $n$-dimensional vector of private asset stocks. A (stationary) Markovian strategy $\phi_{i}$ for player $i$ is a pair of functions $\phi_{i}^{x}:[0,+\infty)^{n+1} \mapsto \mathbb{R}$ and $\phi_{i}^{c}:[0,+\infty)^{n+1} \mapsto \mathbb{R}$. We call $\phi_{i}^{x}$ agent $i$ 's appropriation or extraction strategy and $\phi_{i}^{c}$ his consumption strategy. Applying the strategy $\phi_{i}$ means that agent $i$ chooses his appropriation and consumption rates according to 
the time-invariant feedback laws $x_{i}(t)=\phi_{i}^{x}(\mathbf{y}(t), z(t))$ and $c_{i}(t)=\phi_{i}^{c}(\mathbf{y}(t), z(t))$, respectively. A strategy profile is a $n$-tuple of Markovian strategies, one for each agent. A strategy profile $\left(\phi_{1}, \phi_{2}, \ldots, \phi_{n}\right)$ is called symmetric, if $\phi_{i}=\phi_{j}$ holds for all $i$ and $j$. A strategy profile is a Markov-perfect Nash equilibrium if, for all $z_{0} \in(0,+\infty)$, all $\mathbf{y}_{0}=\left(y_{10}, y_{20}, \ldots, y_{n 0}\right) \in[0,+\infty)^{n}$, and all $i$ the following is true: the optimal control problem of maximizing (5) subject to (1)-(4), $x_{j}(t)=\phi_{j}^{x}(\mathbf{y}(t), z(t))$, and $c_{j}(t)=\phi_{j}^{c}(\mathbf{y}(t), z(t))$ for all $j \neq i$ has an optimal solution which satisfies $x_{i}(t)=\phi_{i}^{x}(\mathbf{y}(t), z(t))$ and $c_{i}(t)=\phi_{i}^{c}(\mathbf{y}(t), z(t))$.

In the above definition we have assumed that Markovian strategies can depend on the common property asset stock and on all private asset stocks. In all equilibria discussed in the present paper, the appropriation and consumption rates of agent $i$ will only depend on the public capital stock and on agent $i$ 's own private asset holdings. Thus, nothing would be lost if one would restrict the domain of admissible strategies in such a way. In particular, it is irrelevant for the results of this paper whether the players can actually observe their opponents' private asset stocks or not.

\section{Main results}

Consider the equation

$$
(\rho-r) u^{\prime}(\bar{q})=w(\bar{q}) .
$$

From the assumptions mentioned in section 2 it follows that the left-hand side decreases strictly from $+\infty$ to 0 as $\bar{q}$ goes from 0 to $+\infty$. The right-hand side is a strictly positive and strictly increasing function of $\bar{q}$. Since both sides depend continuously on $\bar{q}$, there must exist a unique positive solution of equation (6). To understand the relevance of this solution, suppose that the consumption/wealth ratio is constant and equal to $q$. The cost (loss of utility) of reducing consumption at time $t$ by an infinitesimally small amount $\mathrm{d} c$ is $u^{\prime}(q) \mathrm{d} c$. The gain from doing so, on the other hand, is that the private asset holdings at time $t$ increase by $\mathrm{d} c$. This implies that there are $e^{r(\tau-t)} \mathrm{d} c$ additional units of the private asset available at time $\tau \geq t$. The additional discounted utility that can be derived from these additional asset holdings is given by $w(q) \mathrm{d} c /(\rho-r)$. Equation (6) therefore says that, at $q=\bar{q}$, the marginal cost of a reduction of consumption equals its marginal gain. Note that equation (6) defines $\bar{q}$ as a function of $\rho-r$ but that $\bar{q}$ is independent of $\gamma$ and $\kappa$.

Equation (6) may also be written as

$$
r+\frac{w(\bar{q})}{u^{\prime}(\bar{q})}=\rho
$$

which can be interpreted as a wealth-adjusted modified golden rule. Recall that the usual modified golden rule says that the optimal steady-state holdings of a capital stock must be such that the return (marginal productivity) is equal to the rate of time preference. In our model, because wealth appears in the utility function, the rate of return to private capital is $r$ plus the marginal rate of substitution of private capital for consumption which, in turn, is $[\partial U(c, A) / \partial c] /[\partial U(c, A) / \partial y]=w(q) / u^{\prime}(q)$. Here, we have used the fact that $\partial U(c, A) / \partial y=$ 
$\partial U(c, A) / \partial A$ which follows from $A=y+\gamma z$. Note that the wealth-adjusted modified golden rule is not about the steady-state holdings of capital as such, rather it is about the 'holdings' of capital/consumption ratio. It is in fact a no-arbitrage condition.

The no-arbitrage condition (7) involves only the private asset with return $r$. It is reasonable to conjecture that an analogous no-arbitrage condition must hold for the common property asset. Furthermore, because $\gamma$ is the weight given to the common property asset and $\kappa$ is the cost of extracting it, it is intuitively clear that this second condition will depend on the parameters $\gamma$ and $\kappa$. To derive it, let us assume that all agents, except agent $i$, use the appropriation rate $x_{j}(t)=\beta z(t)$, where $\beta$ is a non-negative number. The private return on the common property asset for agent $i$ is then obviously given by $\bar{R}=R-(n-1) \beta$. Since consumption of the resource is not possible without prior extraction, the marginal utility of consumption from the common property asset is given by $[\partial U(c, A) / \partial c]-\kappa=u^{\prime}(q)-\kappa$. Finally, the marginal utility of an additional unit of the common property resource must be $[\partial U(c, A) / \partial z]=\gamma[\partial U(c, A) / \partial A]=$ $\gamma w(q)$. In analogy to equation (7), the no-arbitrage condition for the common property asset should therefore read as

$$
\bar{R}+\frac{\gamma w(\bar{q})}{u^{\prime}(\bar{q})-\kappa}=\rho .
$$

The formal derivation of this condition follows exactly the same logic as that of equation (6) or (7). Suppose that the consumption/wealth ratio is constant and equal to $q$. The cost of reducing appropriation and consumption at time $t$ by an infinitesimally small amount $\mathrm{d} x$ is $\left[u^{\prime}(q)-\kappa\right] \mathrm{d} x$. The gain from doing so is that the common property asset holdings at time $t$ increase by $\mathrm{d} x$. This implies that there are $e^{\bar{R}(\tau-t)} \mathrm{d} x$ additional units of the public asset available at time $\tau \geq t$. The additional discounted utility that can be derived from these additional asset holdings is given by $\gamma w(q) \mathrm{d} x /(\rho-\bar{R})$. Equation (8) therefore says that, at $q=\bar{q}$, the marginal cost of a reduction of appropriation equals its marginal gain.

Let us denote the marginal utility of consumption at $q=\bar{q}$ by $\bar{\kappa}$, that is, $\bar{\kappa}=u^{\prime}(\bar{q})$. Furthermore, provided that $\kappa<\bar{\kappa}$, let us define the number $\bar{\beta}$ by

$$
\bar{\beta}=\frac{1}{n-1}\left[R-\rho+\frac{(\rho-r) \gamma \bar{\kappa}}{\bar{\kappa}-\kappa}\right] .
$$

Using (6) and $\bar{R}=R-(n-1) \beta$ it is easy to see that $\beta=\bar{\beta}$ is the only value of $\beta$ that satisfies the second no-arbitrage condition (8). We are now ready to state the main result of the present section.

Theorem 1 Let $\kappa<\bar{\kappa}$ be given and assume that the condition

$$
\frac{(\rho-r) \gamma \bar{\kappa}}{\bar{\kappa}-\kappa}>\rho-R+(n-1) \gamma \bar{q}
$$

is satisfied. The strategy profile $\left(\phi_{1}, \phi_{2}, \ldots, \phi_{n}\right)$ defined by $\phi_{i}^{c}(\mathbf{y}, z)=\bar{q}\left(y_{i}+\gamma z\right)$ and $\phi_{i}^{x}(\mathbf{y}, z)=\bar{\beta} z$ forms a symmetric Markov-perfect Nash equilibrium.

Before we present the proof of the theorem, it will be useful to derive the following auxiliary result. 
Lemma 1 Let $\kappa<\bar{\kappa}$ be given and assume that condition (10) is satisfied. Then it holds that $\bar{\beta}>\gamma \bar{q}$ and $R-n \bar{\beta}<\rho$.

Proof: Using (9) it is easy to see that the (10) is equivalent to $\bar{\beta}>\gamma \bar{q}$. To prove the second statement of the lemma, we distinguish two cases. If $\rho<R$, then it follows from the assumptions $\kappa<\bar{\kappa}$ and $\rho>r$ that $(\rho-r) \gamma \bar{\kappa} /(\bar{\kappa}-\kappa)>0>(\rho-R) / n$. On the other hand, if $\rho \geq R$, then we obtain from (10) that $(\rho-r) \gamma \bar{\kappa} /(\bar{\kappa}-\kappa)>\rho-R+(n-1) \gamma \bar{q} \geq \rho-R \geq(\rho-R) / n$. In both cases it therefore follows that $(\rho-r) \gamma \bar{\kappa} /(\bar{\kappa}-\kappa)>(\rho-R) / n$. Using (9) again, it is easy to see that the latter condition is equivalent to $R-n \bar{\beta}<\rho . \triangleleft$

Proof of Theorem 1: Suppose that all players $j \neq i$ use the equilibrium strategies $\phi_{j}$. In this situation, player $i$ faces the optimal control problem $P$ defined as follows:

$$
\begin{array}{cl}
\text { maximize } & \int_{0}^{+\infty} e^{-\rho t}\left[U\left(c_{i}(t), y_{i}(t)+\gamma z(t)\right)-\kappa x_{i}(t)\right] \mathrm{d} t \\
\text { subject to } & \dot{z}(t)=\bar{R} z(t)-x_{i}(t) \\
& \dot{y}_{i}(t)=r y_{i}(t)+x_{i}(t)-c_{i}(t) \\
& \dot{y}_{j}(t)=r y_{j}(t)+\bar{\beta} z(t)-\bar{q}\left[y_{j}(t)+\gamma z(t)\right] \text { for } j \neq i \\
& x_{i}(t) \geq 0, c_{i}(t) \geq 0, \mathbf{y}(t) \geq 0, z(t) \geq 0,
\end{array}
$$

where $\bar{R}=R-(n-1) \bar{\beta}$. The Hamiltonian function of this problem is

$$
H=U\left(c_{i}, y_{i}+\gamma z\right)-\kappa x_{i}+\lambda\left(\bar{R} z-x_{i}\right)+\mu_{i}\left(r y_{i}+x_{i}-c_{i}\right)+\sum_{j \neq i} \mu_{j}\left[r y_{j}+\bar{\beta} z-\bar{q}\left(y_{j}+\gamma z\right)\right],
$$

where $\lambda$ and $\mu_{k}, k=1,2, \ldots, n$, are the costate variables corresponding to $z$ and $y_{k}$, respectively. Note that the Hamiltonian is jointly concave in $\left(z, \mathbf{y}, c_{i}, x_{i}\right)$. The theorem is therefore proved if there exist costate trajectories $\lambda:[0,+\infty) \mapsto[0,+\infty)$ and $\mu_{k}:[0,+\infty) \mapsto[0,+\infty), k=$ $1,2, \ldots, n$, such that the feasibility conditions of problem $P$ as well as the following optimality conditions are satisfied by $c_{i}(t)=\bar{q}\left[y_{i}(t)+\gamma z(t)\right]$ and $x_{i}(t)=\bar{\beta} z(t)$ :

$$
\begin{aligned}
& H_{c_{i}}=u^{\prime}\left(c_{i}(t) /\left[y_{i}(t)+\gamma z(t)\right]\right)-\mu_{i}(t)=0 \\
& H_{x_{i}}=\mu_{i}(t)-\lambda(t)-\kappa=0 \\
& \dot{\lambda}(t)=\rho \lambda(t)-H_{z}=(\rho-\bar{R}) \lambda(t)-\gamma w\left(c_{i}(t) /\left[y_{i}(t)+\gamma z(t)\right]\right)-(\bar{\beta}-\gamma \bar{q}) \sum_{j \neq i} \mu_{j}(t), \\
& \dot{\mu}_{i}(t)=\rho \mu_{i}(t)-H_{y_{i}}=(\rho-r) \mu_{i}(t)-w\left(c_{i}(t) /\left[y_{i}(t)+\gamma z(t)\right]\right), \\
& \dot{\mu}_{j}(t)=\rho \mu_{j}(t)-H_{y_{j}}=(\rho-r+\bar{q}) \mu_{j}(t) \\
& \lim _{t \rightarrow+\infty} e^{-\rho t}\left[\lambda(t) z(t)+\sum_{k=1}^{n} \mu_{k}(t) y_{k}(t)\right] \leq 0 .
\end{aligned}
$$

We define the costate trajectories by $\lambda(t)=\bar{\kappa}-\kappa, \mu_{i}(t)=\bar{\kappa}$, and $\mu_{j}(t)=0$ for all $j \neq i$. Note that all costate trajectories are constant and non-negative. Condition (11) holds because of $u^{\prime}(\bar{q})=\bar{\kappa}$ and condition (12) follows immediately from the definitions of $\lambda(t)$ and $\mu_{i}(t)$. Substituting $c_{i}(t) /\left[y_{i}(t)+\gamma z(t)\right]=\bar{q}$ and the definitions of the costate trajectories into (13) we 
obtain $0=(\rho-\bar{R})(\bar{\kappa}-\kappa)-\gamma w(\bar{q})$. This equation holds because of the definition of $\bar{R}$, equation $(9), \bar{\kappa}=u^{\prime}(\bar{q})$, and (6). Analogously, by substituting $c_{i}(t) /\left[y_{i}(t)+\gamma z(t)\right]=\bar{q}$ and $\mu_{i}(t)=\bar{\kappa}$ into (14), one obtains $0=(\rho-r) \bar{\kappa}-w(\bar{q})$. This equation holds because of $\bar{\kappa}=u^{\prime}(\bar{q})$ and $(6)$. Condition (15) holds trivially because of $\mu_{j}(t)=0$ for all $t$.

To verify the transversality condition (16), let us define $g=R-n \bar{\beta}$. Lemma 1 implies that $g<\rho$. From the state equation $\dot{z}(t)=\bar{R} z(t)-x_{i}(t)$ and from $x_{i}(t)=\bar{\beta} z(t)$ it follows that $z(t)=z_{0} e^{g t}$. Consequently, we have $\lim _{t \rightarrow+\infty} e^{-\rho t} \lambda(t) z(t)=(\bar{\kappa}-\kappa) z_{0} \lim _{t \rightarrow+\infty} e^{(g-\rho) t}=0$. From $x_{i}(t)=\bar{\beta} z(t), z(t)=z_{0} e^{g t}$, and the state equation $\dot{y}_{i}(t)=r y_{i}(t)+x_{i}(t)-c_{i}(t)$ we obtain $\dot{y}_{i}(t) \leq r y_{i}(t)+\bar{\beta} z_{0} e^{g t}$. It follows therefore that there exists a positive constant $Y$ such that $y_{i}(t) \leq Y e^{\max \{r, g\} t}$. This implies that $\lim _{t \rightarrow+\infty} e^{-\rho t} \mu_{i}(t) y_{i}(t) \leq \bar{\kappa} Y \lim _{t \rightarrow+\infty} e^{\max \{r-\rho, g-\rho\} t}=0$, whereby the last equation follows from $g<\rho$ and $r<\rho$. Finally, $\lim _{t \rightarrow+\infty} e^{-\rho t} \mu_{j}(t) y_{j}(t)=0$ holds trivially for all $j \neq i$ because $\mu_{j}(t)=0$. The transversality condition (16) is therefore satisfied.

It remains to be shown that the feasibility conditions are not violated. More specifically, we have to show that the application of the strategy $\phi_{i}$ implies that $z(t), y_{i}(t), x_{i}(t)$, and $c_{i}(t)$ remain non-negative for all $t$ (independently of the initial conditions $z_{0}$ and $y_{i 0}$ ). We have already proved that $z(t)=z_{0} e^{g t}$ which shows that, starting from any initial state $z_{0}>0$, the asset stock $z(t)$ remains strictly positive for all $t$. Now note that lemma 1 implies that $\bar{\beta}>\gamma \bar{q}$ and therefore $\bar{\beta}>0$. It follows that $x_{i}(t)=\bar{\beta} z(t) \geq 0$. Application of $\phi_{i}$ implies furthermore that $c_{i}(t)=\bar{q}\left[y_{i}(t)+\gamma z(t)\right]$ and, hence, $\dot{y}_{i}(t)=(\bar{\beta}-\gamma \bar{q}) z(t)+(r-\bar{q}) y_{i}(t)$. This shows that $\left.\dot{y}_{i}(t)\right|_{y_{i}(t)=0}=(\bar{\beta}-\gamma \bar{q}) z(t)>0$, whereby the inequality follows from $z(t)>0$ and $\bar{\beta}>\gamma \bar{q}$ (see lemma 1). Therefore, $y_{i}(t)$ cannot become negative if it starts at a non-negative initial value. Because $c_{i}(t)=\bar{q}\left[y_{i}(t)+\gamma z(t)\right]$, it follows also that $c_{i}(t)$ remains non-negative. This completes the proof of the theorem. $\triangleleft$

The equilibrium described in the above theorem corresponds to the interior equilibrium discussed in the papers by Tornell and Velasco [11] and Tornell and Lane [10]. It is an equilibrium in which players are indifferent about how much to extract from the common property resource. In the proof of theorem 1 this can be seen from the fact that the condition $H_{x_{i}}=0$ holds.

Theorem 1 has two crucial assumptions, namely, $\kappa<\bar{\kappa}$ and condition (10). Note that the latter is satisfied if $R-\rho$ is sufficiently large or if $\gamma>0$ and $\kappa$ is sufficiently close to (but smaller than) $\bar{\kappa}$. Note furthermore that, for $\gamma=0$, condition (10) reduces to the simple inequality $R>\rho$.

A further remark on theorem 1 concerns the relative size of $R$ and $r$. In Tornell and Velasco [11] and Tornell and Lane [10] it is assumed that $R>r$ holds. If $\gamma=0$, this is exactly what condition (10) implies. In the present model, however, the assumption $R>r$ is not necessary. As a matter of fact, let us consider the case where $R$ and $r$ are chosen such that $R<r<\rho$ holds and where $\gamma$ is positive. As has been mentioned before, condition (10) will be satisfied in this situation provided that $\kappa$ is sufficiently close to $\bar{\kappa}$. Thus, the interior Markov-perfect Nash equilibrium described in theorem 1 exists also in situations where the total return on the common property asset, $R$, is strictly smaller than the return on the private asset, $r$.

In Tornell and Velasco [11] and Tornell and Lane [10] it has been shown that, in the interior equilibrium corresponding to the equilibrium from theorem 1, the private rate of return on the 
public asset has to coincide with the rate of return on the private asset. To see whether this holds true in our model, let us compute the private rate of return on the common property asset in the equilibrium from theorem 1. It is given by $\bar{R}=R-(n-1) \bar{\beta}$ which, because of (9), is equal to

$$
\bar{R}=\rho-\frac{(\rho-r) \gamma \bar{\kappa}}{\bar{\kappa}-\kappa}
$$

Tornell and Velasco [11] and Tornell and Lane [10] consider a situation where none of the two asset stocks is an argument of the utility function. This implies of course that both asset stocks are treated equally which corresponds in our model to the case $\gamma=1$. Furthermore, Tornell and Velasco [11] and Tornell and Lane [10] do not consider any appropriation costs which would be reflected in our model by $\kappa=0$. Substituting $\gamma=1$ and $\kappa=0$ into (17) it follows indeed that $\bar{R}=r$. Thus, if we choose $\gamma$ and $\kappa$ such as to make our model as similar as possible to that of Tornell and Velasco [11] and Tornell and Lane [10], we obtain exactly their result. In general, however, $\bar{R}$ can be smaller or larger than $r$, as can be easily shown by inspection of (17). As a matter of fact, $\bar{R}=r$ holds if and only if $\kappa=(1-\gamma) \bar{\kappa}, \bar{R}>r$ holds if and only if $\kappa<(1-\gamma) \bar{\kappa}$, and $\bar{R}<r$ holds if and only if $\kappa>(1-\gamma) \bar{\kappa}$. These results involve the monetary returns $r$ and $\bar{R}$. One could define 'full' rates of return for the two types of assets by the left-hand sides of equation (7) and (8), respectively. In that case, both rates of return would obviously be equal to the time-preference $\rho$ such that return equalization across assets would hold.

Let us now discuss the monotonicity properties of $\bar{R}$ and $\bar{\beta}$ with respect to the model parameters.

Lemma 2 The private rate of return on the public asset, $\bar{R}$ from equation (17), is decreasing with respect to the appropriation cost $\kappa$, strictly decreasing with respect to the weight $\gamma$, and it is independent of the number of players $n$.

Proof: The lemma follows immediately from (17), $\rho>r$, and $\gamma \geq 0 . \triangleleft$

The monotonicity with respect to $\kappa$ can be explained as follows. The interior equilibrium is an equilibrium, in which agents extract the resource more quickly than they consume it. Positive appropriation costs form an impediment to this voracious behavior, because these costs are incurred before the resource is consumed and the agents have a strict time-preference. The agents will only be voracious if the rate of return on the private asset is sufficiently high relative to the return of the public asset. Since the rate of return on the private asset is a fixed constant $r$, this implies that, for high appropriation costs, the rate of return on the common property asset, $\bar{R}$, must be very low. The fact that $\bar{R}$ is strictly decreasing with respect to $\gamma$ is also easy to interpret. A higher value of $\gamma$ means that agents attach less weight to their private asset holdings. Therefore, they have a weaker incentive to be voracious and, hence, the rate of return on the private asset has to be high relative to the rate of return on the public asset in order for voracious behavior to qualify as an equilibrium. This, in turn, means that the private rate of return on the common property asset must be low if $\gamma$ is high. The fact that $\bar{R}$ is independent of $n$ follows immediately from the no-arbitrage condition (8).

Lemma 3 The equilibrium extraction intensity, $\bar{\beta}$ from equation (9), is increasing with respect to the appropriation cost $\kappa$, strictly increasing with respect to the weight $\gamma$, and strictly decreasing 
with respect to the number of players $n$. Furthermore, the aggregate extraction intensity $n \bar{\beta}$ is strictly decreasing with respect to $n$.

Proof: The first part of this lemma follows immediately from lemma 2 and $\bar{R}=R-(n-1) \bar{\beta}$. The second part follows from the facts that $\bar{R}$ is independent of $n$ (see lemma 2) and that $\bar{\beta}$ is strictly decreasing with respect to $n$ (this lemma), and from $n \bar{\beta}=R-\bar{R}+\bar{\beta} . \triangleleft$

The results of this lemma are basically the same as in Tornell and Velasco [11] and Tornell and Lane [10]. Finally, we investigate monotonicity properties of the equilibrium growth rate of the public asset. This growth rate is given by $g=R-n \bar{\beta}$. The following results hold.

Lemma 4 The equilibrium growth rate of the common property asset, $g$, is decreasing with respect to the appropriation cost $\kappa$, strictly decreasing with respect to the weight $\gamma$ and the raw rate of return $R$, and strictly increasing with respect to the number of players $n$.

Proof: From the definition of $g$ and from (9) it follows that

$$
g=\frac{n}{n-1}\left[\rho-\frac{(\rho-r) \gamma \bar{\kappa}}{\bar{\kappa}-\kappa}\right]-\frac{R}{n-1} .
$$

From this expression it is easy to see that the first three monotonicity properties stated in the

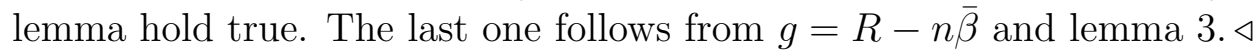

Tornell and Lane [10] call the fact that $g$ decreases with respect to $R$ the voracity effect, which they define as "a more-than-proportional increase in discretionary redistribution in response to an increase in the raw rate of return in the efficient sector" ([10, p. 34]). In our model, the discretionary redistribution corresponds to the term $n \bar{\beta}$. Our model allows also another interesting observation that follows from the monotonicity of $g$ with respect to $\kappa$. More specifically, if one interprets $\kappa$ as the cost of money laundering, then it follows that by reducing the cost of money laundering, a government can increase the net growth rate of the public asset.

Given that both consumption and wealth are arguments of the utility function, it is natural to ask whether the elasticity of substitution between consumption and wealth has any effect on the equilibrium growth rate or the intensity of extraction. In order to address this question we assume that the utility function is of the CES type

$$
U(c, A)=\left(c^{\varepsilon}+A^{\varepsilon}\right)^{1 / \varepsilon},
$$

where $\varepsilon$ is a real number smaller than 1 and different from 0 . The elasticity of substitution is then given by $\sigma=1 /(1-\varepsilon)$. The corresponding functions $u$ and $w$ are given by $u(q)=\left(1+q^{\varepsilon}\right)^{1 / \varepsilon}$ and $w(q)=\left(1+q^{\varepsilon}\right)^{(1-\varepsilon) / \varepsilon}$, respectively. It has to be mentioned that the CES function does not satisfy the Inada conditions in assumption U. Nor does it satisfy the condition $u(0)=0$ when $\varepsilon$ is positive. However, we have used these conditions only to ascertain the existence of a solution to equation (6). Here, we shall show directly that (6) has a unique solution such that the rest of our analysis remains valid. Indeed, it is straightforward to see that equation (6) 
yields $\bar{q}=(\rho-r)^{1 /(1-\varepsilon)}$ and, hence, $\bar{\kappa}=u^{\prime}(\bar{q})=\left[1+(\rho-r)^{-\varepsilon /(1-\varepsilon)}\right]^{(1-\varepsilon) / \varepsilon}$. Combining this with $\sigma=1 /(1-\varepsilon)$ shows that

$$
\bar{\kappa}=\left[1+(\rho-r)^{1-\sigma}\right]^{1 /(\sigma-1)} .
$$

From (9), we have

$$
\frac{\mathrm{d} \bar{\beta}}{\mathrm{d} \bar{\kappa}}=-\frac{(\rho-r) \gamma \kappa}{(n-1)(\bar{\kappa}-\kappa)^{2}}<0
$$

and from (18) follows

$$
\frac{\mathrm{d} \bar{\kappa}}{\mathrm{d} \sigma}=-\bar{\kappa}\left[\frac{\ln \left[1+(\rho-r)^{1-\sigma}\right]}{(\sigma-1)^{2}}+\frac{(\rho-r)^{1-\sigma} \ln (\rho-r)}{(\sigma-1)\left[1+(\rho-r)^{1-\sigma}\right]}\right]
$$

The first term in the square brackets is always positive but the sign of the second term depends on whether $\sigma$ is greater or smaller than 1 and on whether $\rho-r$ is greater or smaller than 1 . If $\sigma>1$ (which is equivalent to $\varepsilon>0$ ) and $\rho-r \geq 1$, then it follows that the second term in brackets is non-negative such that $\mathrm{d} \bar{\kappa} / \mathrm{d} \sigma<0$. This, in turn, implies together with (19) that $\mathrm{d} \bar{\beta} / \mathrm{d} \sigma>0$. We conclude that, under the stated assumptions, a higher elasticity of substitution will lead to higher intensity of extraction.

\section{Heterogeneous players}

So far we have looked at the case of homogeneous players. The present section, on the other hand, discusses the effects of differences between players. To simplify the analysis, we restrict ourselves to case of only two types of players. More specifically, let us assume that there are $n_{1} \geq 1$ players described by the parameters $\left(\rho_{1}, \gamma_{1}, \kappa_{1}, r_{1}\right)$ and the utility function $U_{1}$ and $n_{2} \geq 1$ players described by the parameters $\left(\rho_{2}, \gamma_{2}, \kappa_{2}, r_{2}\right)$ and the utility function $U_{2}$. The total number of players is $n=n_{1}+n_{2}$. We assume that assumptions $\mathrm{P}$ and $\mathrm{U}$ hold for both types. For each $\ell \in\{1,2\}$, we will denote by $u_{\ell}$ and $w_{\ell}$ the marginal utility of consumption $u_{\ell}(q)=U_{\ell}(q, 1)$ and the marginal utility of wealth $w_{\ell}(q)=u_{\ell}(q)-u_{\ell}^{\prime}(q) q$.

In analogy to section 3 , we define $\bar{q}_{\ell}$ as the unique positive number satisfying

$$
\left(\rho_{\ell}-r_{\ell}\right) u_{\ell}^{\prime}\left(\bar{q}_{\ell}\right)=w_{\ell}\left(\bar{q}_{\ell}\right),
$$

and we set $\bar{\kappa}_{\ell}=u_{\ell}^{\prime}\left(\bar{q}_{\ell}\right)$. Furthermore, we define

$$
A_{\ell}=\frac{\left(\rho_{\ell}-r_{\ell}\right) \gamma_{\ell} \bar{\kappa}_{\ell}}{\bar{\kappa}_{\ell}-\kappa_{\ell}}
$$

and $B_{\ell}=R-\rho_{\ell}+A_{\ell}$. Finally, we define

$$
\begin{aligned}
& \bar{\beta}_{1}=\left[B_{1}-\left(B_{1}-B_{2}\right) n_{2}\right] /(n-1), \\
& \bar{\beta}_{2}=\left[B_{2}-\left(B_{2}-B_{1}\right) n_{1}\right] /(n-1) .
\end{aligned}
$$


The following parameter restriction will be crucial for the main result of this section.

$$
\begin{aligned}
& n_{1} A_{1}-\left(n_{1}-1\right) A_{2}>n_{1} \rho_{1}-\left(n_{1}-1\right) \rho_{2}+(n-1) \gamma_{2} \bar{q}_{2}-R, \\
& n_{2} A_{2}-\left(n_{2}-1\right) A_{1}>n_{2} \rho_{2}-\left(n_{2}-1\right) \rho_{1}+(n-1) \gamma_{1} \bar{q}_{1}-R .
\end{aligned}
$$

Let us denote the type of player $j \in\{1,2, \ldots, n\}$ by $\ell(j)$. We are now ready to characterize the interior equilibrium of the game with heterogeneous players.

Theorem 2 Assume that the parameter values satisfy $\kappa_{\ell}<\bar{\kappa}_{\ell}, \ell \in\{1,2$,$\} , as well as condition$ (22). The strategy profile $\left(\phi_{1}, \phi_{2}, \ldots, \phi_{n}\right)$ defined by $\phi_{i}^{c}(\mathbf{y}, z)=\bar{q}_{\ell(i)}\left(y_{i}+\gamma_{\ell(i)} z\right)$ and $\phi_{i}^{x}(\mathbf{y}, z)=$ $\bar{\beta}_{\ell(i)} z$ forms a Markov-perfect Nash equilibrium.

The proof of this theorem is very similar to that of theorem 1, which is why we do not present it here. ${ }^{5}$

Theorem 2 can be used to study the influence of heterogeneity on the equilibrium outcome. As an illustration, we discuss the effect of differences in appropriation costs on the equilibrium growth rate of the public asset. Suppose that $\kappa_{1}=\hat{\kappa}+\varepsilon / n_{1}$ and $\kappa_{2}=\hat{\kappa}-\varepsilon / n_{2}$, where $\hat{\kappa}$ and $\varepsilon$ are real numbers such that $n_{2}\left(\hat{\kappa}-\bar{\kappa}_{2}\right)<\varepsilon<n_{1}\left(\bar{\kappa}_{1}-\hat{\kappa}\right)$. The latter condition ensures that $\kappa_{\ell}<\bar{\kappa}_{\ell}$ holds for each $\ell \in\{1,2$,$\} , as required by theorem 2. An increase in |\varepsilon|$ corresponds to a mean-preserving spread in the distribution of extraction costs across players. Defining

$$
W=\left[\frac{\left(\rho_{2}-r_{2}\right) \gamma_{2} \bar{\kappa}_{2}}{\left(\rho_{1}-r_{1}\right) \gamma_{1} \bar{\kappa}_{1}}\right]^{1 / 2}
$$

we have the following result

Lemma 5 Suppose that $\gamma_{1}$ and $\gamma_{2}$ are strictly positive. The equilibrium growth rate of the common property asset, $g=R-n_{1} \bar{\beta}_{1}-n_{2} \bar{\beta}_{2}$, is a strictly concave function of $\varepsilon$ which attains its unique maximum at

$$
\varepsilon^{*}=\frac{n_{1} n_{2}\left[(1-W) \hat{\kappa}+W \bar{\kappa}_{1}-\bar{\kappa}_{2}\right]}{n_{1}+n_{2} W} .
$$

Proof: From the definitions of $\bar{\beta}_{1}$ and $\bar{\beta}_{2}$ we have

$$
g=R-\frac{n_{1} B_{1}+n_{2} B_{2}}{n-1}=-\frac{R+n_{1}\left(A_{1}-\rho_{1}\right)+n_{2}\left(A_{2}-\rho_{2}\right)}{n-1} .
$$

It is therefore sufficient to prove that $f(\varepsilon)=n_{1}\left(A_{1}-\rho_{1}\right)+n_{2}\left(A_{2}-\rho_{2}\right)$ is strictly convex with respect to $\varepsilon$ and that it attains its minimum at $\varepsilon=\varepsilon^{*}$. Using the definitions of $A_{1}$ and $A_{2}$ we obtain

$$
f^{\prime}(\varepsilon)=\frac{\left(\rho_{1}-r_{1}\right) \gamma_{1} \bar{\kappa}_{1}}{\left(\bar{\kappa}_{1}-\hat{\kappa}-\varepsilon / n_{1}\right)^{2}}-\frac{\left(\rho_{2}-r_{2}\right) \gamma_{2} \bar{\kappa}_{2}}{\left(\bar{\kappa}_{2}-\hat{\kappa}+\varepsilon / n_{2}\right)^{2}}
$$

\footnotetext{
${ }^{5}$ The proof is available from the authors upon request.
} 
and

$$
f^{\prime \prime}(\varepsilon)=\frac{2\left(\rho_{1}-r_{1}\right) \gamma_{1} \bar{\kappa}_{1}}{n_{1}\left(\bar{\kappa}_{1}-\hat{\kappa}-\varepsilon / n_{1}\right)^{3}}+\frac{2\left(\rho_{2}-r_{2}\right) \gamma_{2} \bar{\kappa}_{2}}{n_{2}\left(\bar{\kappa}_{2}-\hat{\kappa}+\varepsilon / n_{2}\right)^{3}} .
$$

Obviously, we have $f^{\prime \prime}(\varepsilon)>0$ for all $\varepsilon \in\left(n_{2}\left(\hat{\kappa}-\bar{\kappa}_{2}\right), n_{1}\left(\bar{\kappa}_{1}-\hat{\kappa}\right)\right)$ and $f^{\prime}(\varepsilon)=0$ if and only if $\varepsilon=\varepsilon^{*}$ This completes the proof of the lemma. $\triangleleft$

As a special case, suppose that the cost parameters are the only source of heterogeneity. Formally, let us assume that the conditions $\gamma_{1}=\gamma_{2}, r_{1}=r_{2}, \bar{\kappa}_{1}=\bar{\kappa}_{2}$, and $\rho_{1}=\rho_{2}$ hold. In this case, $W=1$ and $\varepsilon^{*}=0$. We therefore conclude that, in a model in which the appropriation cost is the only source of heterogeneity, a mean-preserving spread in the distribution of this cost across players leads to a reduction of the equilibrium growth rate of the public asset. On the other hand, if players differ also in other characteristics, then it holds that $\varepsilon^{*} \neq 0$ and that there exists a non-zero degree of cost heterogeneity that maximizes the net growth rate of the public asset.

\section{The pessimistic equilibrium}

In this section we assume that the players have to obey the additional constraint

$$
x_{i}(t) \leq \beta_{H} z(t)
$$

for all $t$ and all $i$, where $\beta_{H}$ is a given positive constant. In other words, each player's extraction intensity must not exceed the exogenously given upper bound $\beta_{H}$. Such a constraint may interpreted, for example, as a certain form of capital control. If $\beta_{H} \geq \bar{\beta}$ and the conditions of theorem 1 are satisfied, then the strategy profile described in that theorem remains to qualify as a Markov-perfect Nash equilibrium. However, there will also exists another equilibrium along which all agents extract the common property asset at maximum speed. This is proved in the following theorem.

Theorem 3 Consider the differential game specified by (1)-(5) and (23). Let $\kappa<\bar{\kappa}$ be given and assume that the inequalities $\beta_{H}>\gamma \bar{q}$ and

$$
\frac{(\rho-r) \gamma \bar{\kappa}}{\bar{\kappa}-\kappa} \leq \rho-R+(n-1) \beta_{H}
$$

are satisfied. The strategy profile $\left(\phi_{1}, \phi_{2}, \ldots, \phi_{n}\right)$ defined by $\phi_{i}^{c}(\mathbf{y}, z)=\bar{q}\left(y_{i}+\gamma z\right)$ and $\phi_{i}^{x}(\mathbf{y}, z)=$ $\beta_{H} z$ forms a symmetric Markov-perfect Nash equilibrium.

Before we prove this theorem we state the following auxiliary result.

Lemma 6 Let $\kappa<\bar{\kappa}$ be given and assume that (24) holds. Then it follows that $\beta_{H}>(R-\rho) / n$. 
Proof: Because of (24), $\gamma \geq 0, \rho>r, \kappa<\bar{\kappa}$, and $\beta_{H}>0$ it holds that

$$
\rho-R+n \beta_{H} \geq \beta_{H}+\frac{(\rho-r) \gamma \bar{\kappa}}{\bar{\kappa}-\kappa}>0 .
$$

This proves the lemma. $\triangleleft$

Proof of Theorem 3: Suppose that all players $j \neq i$ use the equilibrium strategies $\phi_{j}$. In this situation, player $i$ faces the following optimal control problem $Q$ :

$$
\begin{array}{cl}
\operatorname{maximize} & \int_{0}^{+\infty} e^{-\rho t}\left[U\left(c_{i}(t), y_{i}(t)+\gamma z(t)\right)-\kappa x_{i}(t)\right] \mathrm{d} t \\
\text { subject to } & \dot{z}(t)=\tilde{R} z(t)-x_{i}(t) \\
& \dot{y}_{i}(t)=r y_{i}(t)+x_{i}(t)-c_{i}(t) \\
& \dot{y}_{j}(t)=r y_{j}(t)+\beta_{H} z(t)-\bar{q}\left[y_{j}(t)+\gamma z(t)\right] \text { for } j \neq i \\
& 0 \leq x_{i}(t) \leq \beta_{H} z(t), c_{i}(t) \geq 0, \mathbf{y}(t) \geq 0, z(t) \geq 0,
\end{array}
$$

where $\tilde{R}=R-(n-1) \beta_{H}$. The Lagrangian function of this problem is

$$
L=U\left(c_{i}, y_{i}+\gamma z\right)-\kappa x_{i}+\lambda\left(\tilde{R} z-x_{i}\right)+\mu_{i}\left(r y_{i}+x_{i}-c_{i}\right)+\sum_{j \neq i} \mu_{j}\left[r y_{j}+\beta_{H} z-\bar{q}\left(y_{j}+\gamma z\right)\right]+\nu\left(\beta_{H} z-x_{i}\right),
$$

where $\lambda$ and $\mu_{k}, k=1,2, \ldots, n$, have the same interpretation as in the proof of theorem 1 and where $\nu$ is the Lagrange multiplier for (23). Note that the Lagrangian is jointly concave in $\left(z, \mathbf{y}, c_{i}, x_{i}\right)$. The theorem is therefore proved if there exist costate trajectories $\lambda:[0,+\infty) \mapsto$ $[0,+\infty)$ and $\mu_{k}:[0,+\infty) \mapsto[0,+\infty), k=1,2, \ldots, n$, as well as a multiplier $\nu:[0,+\infty) \mapsto$ $[0,+\infty)$ such that the feasibility conditions of problem $Q$ as well as the following optimality conditions are satisfied by $c_{i}(t)=\bar{q}\left[y_{i}(t)+\gamma z(t)\right]$ and $x_{i}(t)=\beta_{H} z(t)$ :

$$
\begin{aligned}
& u^{\prime}\left(c_{i}(t) /\left[y_{i}(t)+\gamma z(t)\right]\right)-\mu_{i}(t)=0, \\
& \mu_{i}(t)-\lambda(t)-\kappa-\nu(t)=0, \\
& \dot{\lambda}(t)=(\rho-\tilde{R}) \lambda(t)-\gamma w\left(c_{i}(t) /\left[y_{i}(t)+\gamma z(t)\right]\right)-\left(\beta_{H}-\gamma \bar{q}\right) \sum_{j \neq i} \mu_{j}(t)-\beta_{H} \nu(t), \\
& \dot{\mu}_{i}(t)=(\rho-r) \mu_{i}(t)-w\left(c_{i}(t) /\left[y_{i}(t)+\gamma z(t)\right]\right), \\
& \dot{\mu}_{j}(t)=(\rho-r+\bar{q}) \mu_{j}(t) \\
& \nu(t)\left[\beta_{H} z(t)-x_{i}(t)\right]=0 \\
& \lim _{t \rightarrow+\infty} e^{-\rho t}\left[\lambda(t) z(t)+\sum_{k=1}^{n} \mu_{k}(t) y_{k}(t)\right] \leq 0 .
\end{aligned}
$$

We define the costate trajectories by

$$
\begin{aligned}
& \lambda(t)=\frac{\gamma(\rho-r) \bar{\kappa}+\beta_{H}(\bar{\kappa}-\kappa)}{\rho-R+n \beta_{H}}, \\
& \mu_{i}(t)=\bar{\kappa}, \\
& \mu_{j}(t)=0 \text { for } j \neq i .
\end{aligned}
$$


Because of $\beta_{H}>(R-\rho) / n$ (see lemma 6$)$ and $\kappa<\bar{\kappa}$ it follows that all costate trajectories are constant and non-negative. The Lagrange multiplier $\nu$ is defined by

$$
\nu(t)=\frac{(\bar{\kappa}-\kappa)\left[\rho-R+(n-1) \beta_{H}\right]-\gamma(\rho-r) \bar{\kappa}}{\rho-R+n \beta_{H}} .
$$

Because of lemma 6) and (24) we have $\nu(t) \geq 0$. Using these definitions and (6), it is straightforward to verify (25)-(29). Condition (30) holds because of $x_{i}(t)=\beta_{H} z(t)$.

Defining $g_{H}$ by $g_{H}=R-n \beta_{H}$, it follows from lemma 6) that $g_{H}<\rho$. The transversality condition (31) can now be verified in exactly the same way as in the proof of theorem 1 . Finally, we have to show that the application of the strategy $\phi_{i}$ implies that $z(t), y_{i}(t), x_{i}(t)$, and $c_{i}(t)$ remain non-negative for all $t$. Because $\beta_{H}>\gamma \bar{q}$ has been assumed, this property can be verified in essentially the same way as in the proof of theorem 1 . This completes the proof of the theorem. $\triangleleft$

If the assumptions of theorem 1 and $\beta_{H} \geq \bar{\beta}$ hold, then the condition $\beta_{H}>\gamma \bar{q}$ required in theorem 3 is satisfied. This follows trivially from lemma 1. Moreover, condition (24) is true provided that $\beta_{H}$ is sufficiently large. The assumptions of theorem 3 are therefore satisfied whenever those of theorem 1 hold and when $\beta_{H}$ is sufficiently large. It follows that in this case the pessimistic equilibrium from theorem 3 and the interior equilibrium from theorem 1 coexist. The equilibrium stated in theorem 3 does not exist if extraction intensities are not bounded above, that is, if the constraint (23) is missing. If one would introduce a similar lower bound, that is a constraint of the form $x_{i}(t) \geq \beta_{L} z(t)$, then it would be possible to derive also an optimistic equilibrium, in which all players extract with intensity $\beta_{L}$. The structure of the equilibrium strategies in this case would be much more complicated because of the co-existence of the constraints $x_{i}(t) \geq \beta_{L} z(t)$ and $y_{i}(t) \geq 0$. In particular, we believe that the strategies of player $i$ in any Markov-perfect Nash equilibrium would have to depend non-trivially on all private asset stocks and not only on the own private asset holdings $y_{i} \cdot{ }^{6}$ We therefore leave the characterization of the optimistic equilibrium for a separate paper.

\section{Concluding remarks}

In this paper, we have shown that new insights can be gained by adding appropriation costs and wealth effects to the model developed by Tornell and Velasco [11] and Tornell and Lane [10]. A number of testable implications have been derived. For example, countries where powerful groups have equal appropriation costs have higher growth rates than countries where powerful groups have unequal costs. Furthermore, an increase in the cost of money laundering reduces growth.

Our model can be extended in several directions. One may suppose that agents care about both relative wealth and absolute wealth. This would make the status-seeking motive for the agents

\footnotetext{
${ }^{6}$ Tornell and Velasco [11] characterize optimistic equilibria in a model without non-negativity constraints on private asset holdings.
} 
even stronger than in the present model. One could also model status-seeking by making the utility function dependent on relative consumption. These extensions, as well as others, are part of our future research agenda. 


\section{References}

[1] H. Cole, G. Mailath, and A. Postlewaite: Social norms, savings behavior, and growth, Journal of Political Economy 100 (1992) 1092-1125.

[2] J. Dutta and C. Rowat: The road to extinction: commons with capital markets, Working Paper, Department of Economics, University of Birmingham, 2004.

[3] G. Gaudet, M. Moreaux, and S. Salant: Private storage of common property, Journal of Environmental Economics and Management 43 (2002), 280-302.

[4] M. Kremer and C. Morcom: Elephants, American Economic Review 90 (2000), 212-234.

[5] P. Lane and A. Tornell: Power, growth, and the voracity effect, Journal of Economic Growth 1 (1996), 213-241.

[6] I. Lindner and H. Strulik: Why not Africa? - On growth and welfare effects of secure property rights, Public Choice (forthcoming). [http://www.rrz.unihamburg.de/holler/pdf/papers/Lindner_Strulik_Africa.pdf]

[7] H.-W. Sinn: Common property resources, storage facilities and ownership structures: a Cournot model of the oil market, Economica 51 (1984), 235-252.

[8] G. Sorger: A dynamic common property resource problem with amenity value and extraction costs, Working Paper, Department of Economics, University of Vienna, 2004.

[9] A. Tornell: Economic growth and decline with endogenous property rights, Journal of Economic Growth 2 (1997), 219-250.

[10] A. Tornell and P. Lane: The voracity effect, American Economic Review 89 (1999), 22-46.

[11] A. Tornell and A. Velasco: The tragedy of the commons and economic growth: why does capital flow from poor to rich countries?, Journal of Political Economy 100 (1992), 12081231. 


\section{Appendix (available upon request)}

This appendix contains the proof of theorem 2. We start with the following auxiliary result.

Lemma 7 Assume that the parameter values satisfy $\kappa_{\ell}<\bar{\kappa}_{\ell}, \ell \in\{1,2$,$\} , as well as$ condition (22). Then it holds that $\bar{\beta}_{\ell}>\gamma_{\ell} \bar{q}_{\ell}$ and $R-n_{1} \bar{\beta}_{1}-n_{2} \bar{\beta}_{2}<\min \left\{\rho_{1}, \rho_{2}\right\}$.

Proof: Using the definitions of $\bar{\beta}_{1}$ and $\bar{\beta}_{\ell}$ it is straightforward to verify that the two inequalities in (22) are equivalent to $\bar{\beta}_{1}>\gamma_{1} \bar{q}_{1}$ and $\bar{\beta}_{2}>\gamma_{2} \bar{q}_{2}$, respectively. Furthermore, because of the first inequality in $(22), \gamma_{1} \geq 0$, and $A_{1} \geq 0$ we have

$$
\begin{aligned}
n_{2} \rho_{2}-\left(n_{2}-1\right) \rho_{1}-R & <n_{2} A_{2}-\left(n_{2}-1\right) A_{1}-(n-1) \gamma_{1} \bar{q}_{1} \\
& \leq n_{2} A_{2}-\left(n_{2}-1\right) A_{1} \\
& =n_{2} A_{2}+n_{1} A_{1}-(n-1) A_{1} \\
& \leq n_{2} A_{2}+n_{1} A_{1} .
\end{aligned}
$$

Using the definitions of $\bar{\beta}_{1}$ and $\bar{\beta}_{\ell}$ it is easy to verify that this condition is equivalent to $R-n_{1} \bar{\beta}_{1}-n_{2} \bar{\beta}_{2}<\rho_{1}$. The inequality $R-n_{1} \bar{\beta}_{1}-n_{2} \bar{\beta}_{2}<\rho_{2}$ can be proved in analogous way by starting from the second inequality in (22) instead of the first one. This completes the proof of the lemma. $\triangleleft$

Proof of Theorem 2: Without loss of generality we may assume that player $i$ is of type 1. If all players $j \neq i$ use their equilibrium strategies $\phi_{j}$, then it follows that player $i$ faces the following optimal control problem $P_{1}$ :

$$
\begin{array}{cl}
\operatorname{maximize} & \int_{0}^{+\infty} e^{-\rho_{1} t}\left[U_{1}\left(c_{i}(t), y_{i}(t)+\gamma_{1} z(t)\right)-\kappa_{1} x_{i}(t)\right] \mathrm{d} t \\
\text { subject to } & \dot{z}(t)=\bar{R} z(t)-x_{i}(t) \\
& \dot{y}_{i}(t)=r_{1} y_{i}(t)+x_{i}(t)-c_{i}(t) \\
& \dot{y}_{j}(t)=r_{\ell(j)} y_{j}(t)+\bar{\beta}_{\ell(j)} z(t)-\bar{q}_{\ell(j)}\left[y_{j}(t)+\gamma_{\ell(j)} z(t)\right] \text { for } j \neq i \\
& x_{i}(t) \geq 0, c_{i}(t) \geq 0, \mathbf{y}(t) \geq 0, z(t) \geq 0
\end{array}
$$

where $\bar{R}_{1}=R-\left(n_{1}-1\right) \bar{\beta}_{1}-n_{2} \bar{\beta}_{2}$. The Hamiltonian function of this problem is

$$
\begin{aligned}
H= & U_{1}\left(c_{i}, y_{i}+\gamma_{1} z\right)-\kappa_{1} x_{i}+\lambda\left(\bar{R}_{1} z-x_{i}\right)+\mu_{i}\left(r_{1} y_{i}+x_{i}-c_{i}\right) \\
& +\sum_{j \neq i} \mu_{j}\left[r_{\ell(j)} y_{j}+\bar{\beta}_{\ell(j)} z-\bar{q}_{\ell(j)}\left(y_{j}+\gamma_{\ell(j)} z\right)\right],
\end{aligned}
$$

where $\lambda$ and $\mu_{k}, k=1,2, \ldots, n$, are the costate variables corresponding to $z$ and $y_{k}$, respectively. Note that the Hamiltonian is jointly concave in $\left(z, \mathbf{y}, c_{i}, x_{i}\right)$. The theorem is therefore proved if there exist costate trajectories $\lambda:[0,+\infty) \mapsto[0,+\infty)$ and $\mu_{k}$ : $[0,+\infty) \mapsto[0,+\infty), k=1,2, \ldots, n$, such that the feasibility conditions of problem $P_{1}$ as 
well as the following optimality conditions are satisfied by $c_{i}(t)=\bar{q}_{1}\left[y_{i}(t)+\gamma_{1} z(t)\right]$ and $x_{i}(t)=\bar{\beta}_{1} z(t)$ :

$$
\begin{aligned}
& u_{1}^{\prime}\left(c_{i}(t) /\left[y_{i}(t)+\gamma_{1} z(t)\right]\right)-\mu_{i}(t)=0 \\
& \mu_{i}(t)-\lambda(t)-\kappa_{1}=0 \\
& \dot{\lambda}(t)=\left(\rho_{1}-\bar{R}_{1}\right) \lambda(t)-\gamma_{1} w_{1}\left(c_{i}(t) /\left[y_{i}(t)+\gamma_{1} z(t)\right]\right)-\sum_{j \neq i} \mu_{j}(t)\left[\bar{\beta}_{\ell(j)}-\gamma_{\ell(j)} \bar{q}_{\ell(j)}\right] \\
& \dot{\mu}_{i}(t)=\left(\rho_{1}-r_{1}\right) \mu_{i}(t)-w_{1}\left(c_{i}(t) /\left[y_{i}(t)+\gamma_{1} z(t)\right]\right), \\
& \dot{\mu}_{j}(t)=\left(\rho_{1}-r_{\ell(j)}+\bar{q}_{\ell(j)}\right) \mu_{j}(t) \\
& \lim _{t \rightarrow+\infty} e^{-\rho_{1} t}\left[\lambda(t) z(t)+\sum_{k=1}^{n} \mu_{k}(t) y_{k}(t)\right] \leq 0
\end{aligned}
$$

We define the costate trajectories by $\lambda(t)=\bar{\kappa}_{1}-\kappa_{1}, \mu_{i}(t)=\bar{\kappa}_{1}$, and $\mu_{j}(t)=0$ for all $j \neq i$. Note that all costate trajectories are constant and non-negative. Condition (32) holds because of $u_{1}^{\prime}\left(\bar{q}_{1}\right)=\bar{\kappa}_{1}$ and condition (33) follows immediately from the definitions of $\lambda(t)$ and $\mu_{i}(t)$. Substituting $c_{i}(t) /\left[y_{i}(t)+\gamma_{1} z(t)\right]=\bar{q}_{1}$ and the definitions of the costate trajectories into (34) we obtain $0=\left(\rho_{1}-\bar{R}_{1}\right)\left(\bar{\kappa}_{1}-\kappa_{1}\right)-\gamma_{1} w_{1}\left(\bar{q}_{1}\right)$. This equation holds because of the definition of $\bar{R}_{1}$, equation (21), $\bar{\kappa}_{1}=u_{1}^{\prime}\left(\bar{q}_{1}\right)$, and (20). Analogously, by substituting $c_{i}(t) /\left[y_{i}(t)+\gamma_{1} z(t)\right]=\bar{q}_{1}$ and $\mu_{i}(t)=\bar{\kappa}_{1}$ into (35), one obtains $0=\left(\rho_{1}-\right.$ $\left.r_{1}\right) \bar{\kappa}_{1}-w_{1}\left(\bar{q}_{1}\right)$. This equation holds because $\bar{\kappa}_{1}=u_{1}^{\prime}\left(\bar{q}_{1}\right)$ and because of (20). Condition (36) holds trivially because of $\mu_{j}(t)=0$ for all $t$.

To verify the transversality condition (37), let us define $g=R-n_{1} \bar{\beta}_{1}-n_{2} \bar{\beta}_{2}$. Lemma 7 implies that $g<\rho_{1}$. From the state equation $\dot{z}(t)=\bar{R}_{1} z(t)-x_{i}(t)$ and from $x_{i}(t)=\bar{\beta}_{1} z(t)$ it follows that $z(t)=z_{0} e^{g t}$. Consequently, we have $\lim _{t \rightarrow+\infty} e^{-\rho_{1} t} \lambda(t) z(t)=\left(\bar{\kappa}_{1}-\right.$ $\left.\kappa_{1}\right) z_{0} \lim _{t \rightarrow+\infty} e^{\left(g-\rho_{1}\right) t}=0$. From $x_{i}(t)=\beta_{1} z(t), z(t)=z_{0} e^{g t}$, and the state equation $\dot{y}_{i}(t)=r_{1} y_{i}(t)+x_{i}(t)-c_{i}(t)$ we obtain $\dot{y}_{i}(t) \leq r_{1} y_{i}(t)+\bar{\beta}_{1} z_{0} e^{g t}$. It follows therefore that there exists a positive constant $Y$ such that $y_{i}(t) \leq Y e^{\max \left\{r_{1}, g\right\} t}$. This implies that $\lim _{t \rightarrow+\infty} e^{-\rho_{1} t} \mu_{i}(t) y_{i}(t) \leq \bar{\kappa}_{1} Y \lim _{t \rightarrow+\infty} e^{\max \left\{r_{1}-\rho_{1}, g-\rho_{1}\right\} t}=0$, whereby the last equation follows from $g<\rho_{1}$ and $r_{1}<\rho_{1}$. Finally, $\lim _{t \rightarrow+\infty} e^{-\rho_{1} t} \mu_{j}(t) y_{j}(t)=0$ holds trivially for all $j \neq i$ because $\mu_{j}(t)=0$. The transversality condition (37) is therefore satisfied.

It remains to be shown that the feasibility conditions are not violated. More specifically, we have to show that the application of the strategy $\phi_{i}$ implies that $z(t), y_{i}(t), x_{i}(t)$, and $c_{i}(t)$ remain non-negative for all $t$ (independently of the initial conditions $z_{0}$ and $y_{i 0}$ ). We have already proved that $z(t)=z_{0} e^{g t}$ which shows that, starting from any initial state $z_{0}>0$, the asset stock $z(t)$ remains strictly positive for all $t$. Now note that lemma 7 implies that $\bar{\beta}_{1}>\gamma_{1} \bar{q}_{1}$ and therefore $\bar{\beta}_{1}>0$. It follows that $x_{i}(t)=\beta_{1} z(t) \geq 0$. Application of $\phi_{i}$ implies furthermore that $c_{i}(t)=\bar{q}_{1}\left[y_{i}(t)+\gamma_{1} z(t)\right]$ and, hence, $\dot{y}_{i}(t)=\left[\bar{\beta}_{1}-\gamma_{1} \bar{q}_{1}\right] z(t)+\left[r_{1}-\bar{q}_{1}\right] y_{i}(t)$. This shows that $\left.\dot{y}_{i}(t)\right|_{y_{i}(t)=0}=\left[\beta_{1}-\gamma_{1} \bar{q}_{1}\right] z(t)>0$, whereby the inequality follows from $z(t)>0$ and $\bar{\beta}_{1}>\gamma_{1} \bar{q}_{1}$ (see lemma 7 ). Therefore, $y_{i}(t)$ cannot become negative if it starts at a non-negative initial value. Because $c_{i}(t)=\bar{q}_{1}\left[y_{i}(t)+\gamma_{1} z(t)\right]$, it follows also that $c_{i}(t)$ remains non-negative. This completes the proof of the theorem. $\triangleleft$ 


\section{CESifo Working Paper Series}

(for full list see www.cesifo.de)

1189 Robert J. Gary-Bobo and Alain Trannoy, Efficient Tuition Fees, Examinations, and Subsidies, May 2004

1190 Saku Aura and Gregory D. Hess, What's in a Name?, May 2004

1191 Sjur Didrik Flåm and Yuri Ermoliev, Investment Uncertainty, and Production Games, May 2004

1192 Yin-Wong Cheung and Jude Yuen, The Suitability of a Greater China Currency Union, May 2004

1193 Inés Macho-Stadler and David Pérez-Castrillo, Optimal Enforcement Policy and Firms' Emissions and Compliance with Environmental Taxes, May 2004

1194 Paul De Grauwe and Marianna Grimaldi, Bubbles and Crashes in a Behavioural Finance Model, May 2004

1195 Michel Berne and Gérard Pogorel, Privatization Experiences in France, May 2004

1196 Andrea Galeotti and José Luis Moraga-González, A Model of Strategic Targeted Advertising, May 2004

1197 Hans Gersbach and Hans Haller, When Inefficiency Begets Efficiency, May 2004

1198 Saku Aura, Estate and Capital Gains Taxation: Efficiency and Political Economy Consideration, May 2004

1199 Sandra Waller and Jakob de Haan, Credibility and Transparency of Central Banks: New Results Based on Ifo's World Economicy Survey, May 2004

1200 Henk C. Kranendonk, Jan Bonenkamp, and Johan P. Verbruggen, A Leading Indicator for the Dutch Economy - Methodological and Empirical Revision of the CPB System, May 2004

1201 Michael Ehrmann, Firm Size and Monetary Policy Transmission - Evidence from German Business Survey Data, May 2004

1202 Thomas A. Knetsch, Evaluating the German Inventory Cycle - Using Data from the Ifo Business Survey, May 2004

1203 Stefan Mittnik and Peter Zadrozny, Forecasting Quarterly German GDP at Monthly Intervals Using Monthly IFO Business Conditions Data, May 2004 
1204 Elmer Sterken, The Role of the IFO Business Climate Indicator and Asset Prices in German Monetary Policy, May 2004

1205 Jan Jacobs and Jan-Egbert Sturm, Do Ifo Indicators Help Explain Revisions in German Industrial Production?, May 2004

1206 Ulrich Woitek, Real Wages and Business Cycle Asymmetries, May 2004

1207 Burkhard Heer and Alfred Maußner, Computation of Business Cycle Models: A Comparison of Numerical Methods, June 2004

1208 Costas Hadjiyiannis, Panos Hatzipanayotou, and Michael S. Michael, Pollution and Capital Tax Competition within a Regional Block, June 2004

1209 Stephan Klasen and Thorsten Nestmann, Population, Population Density, and Technological Change, June 2004

1210 Wolfgang Ochel, Welfare Time Limits in the United States - Experiences with a New Welfare-to-Work Approach, June 2004

1211 Luis H. R. Alvarez and Erkki Koskela, Taxation and Rotation Age under Stochastic Forest Stand Value, June 2004

1212 Bernard M. S. van Praag, The Connexion Between Old and New Approaches to Financial Satisfaction, June 2004

1213 Hendrik Hakenes and Martin Peitz, Selling Reputation When Going out of Business, June 2004

1214 Heikki Oksanen, Public Pensions in the National Accounts and Public Finance Targets, June 2004

1215 Ernst Fehr, Alexander Klein, and Klaus M. Schmidt, Contracts, Fairness, and Incentives, June 2004

1216 Amihai Glazer, Vesa Kanniainen, and Panu Poutvaara, Initial Luck, Status-Seeking and Snowballs Lead to Corporate Success and Failure, June 2004

1217 Bum J. Kim and Harris Schlesinger, Adverse Selection in an Insurance Market with Government-Guaranteed Subsistence Levels, June 2004

1218 Armin Falk, Charitable Giving as a Gift Exchange - Evidence from a Field Experiment, June 2004

1219 Rainer Niemann, Asymmetric Taxation and Cross-Border Investment Decisions, June 2004

1220 Christian Holzner, Volker Meier, and Martin Werding, Time Limits on Welfare Use under Involuntary Unemployment, June 2004 
1221 Michiel Evers, Ruud A. de Mooij, and Herman R. J. Vollebergh, Tax Competition under Minimum Rates: The Case of European Diesel Excises, June 2004

1222 S. Brock Blomberg and Gregory D. Hess, How Much Does Violence Tax Trade?, June 2004

1223 Josse Delfgaauw and Robert Dur, Incentives and Workers' Motivation in the Public Sector, June 2004

1224 Paul De Grauwe and Cláudia Costa Storti, The Effects of Monetary Policy: A MetaAnalysis, June 2004

1225 Volker Grossmann, How to Promote R\&D-based Growth? Public Education Expenditure on Scientists and Engineers versus R\&D Subsidies, June 2004

1226 Bart Cockx and Jean Ries, The Exhaustion of Unemployment Benefits in Belgium. Does it Enhance the Probability of Employment?, June 2004

1227 Bertil Holmlund, Sickness Absence and Search Unemployment, June 2004

1228 Klaas J. Beniers and Robert Dur, Politicians' Motivation, Political Culture, and Electoral Competition, June 2004

1229 M. Hashem Pesaran, General Diagnostic Tests for Cross Section Dependence in Panels, July 2004

1230 Wladimir Raymond, Pierre Mohnen, Franz Palm, and Sybrand Schim van der Loeff, An Empirically-Based Taxonomy of Dutch Manufacturing: Innovation Policy Implications, July 2004

1231 Stefan Homburg, A New Approach to Optimal Commodity Taxation, July 2004

1232 Lorenzo Cappellari and Stephen P. Jenkins, Modelling Low Pay Transition Probabilities, Accounting for Panel Attrition, Non-Response, and Initial Conditions, July 2004

1233 Cheng Hsiao and M. Hashem Pesaran, Random Coefficient Panel Data Models, July 2004

1234 Frederick van der Ploeg, The Welfare State, Redistribution and the Economy, Reciprocal Altruism, Consumer Rivalry and Second Best, July 2004

1235 Thomas Fuchs and Ludger Woessmann, What Accounts for International Differences in Student Performance? A Re-Examination Using PISA Data, July 2004

1236 Pascalis Raimondos-Møller and Alan D. Woodland, Measuring Tax Efficiency: A Tax Optimality Index, July 2004

1237 M. Hashem Pesaran, Davide Pettenuzzo, and Allan Timmermann, Forecasting Time Series Subject to Multiple Structural Breaks, July 2004 
1238 Panu Poutvaara and Andreas Wagener, The Invisible Hand Plays Dice: Eventualities in Religious Markets, July 2004

1239 Eckhard Janeba, Moral Federalism, July 2004

1240 Robert S. Chirinko, Steven M. Fazzari, and Andrew P. Meyer, That Elusive Elasticity: A Long-Panel Approach to Estimating the Capital-Labor Substitution Elasticity, July 2004

1241 Hans Jarle Kind, Karen Helene Midelfart, Guttorm Schjelderup, Corporate Tax Systems, Multinational Enterprises, and Economic Integration, July 2004

1242 Vankatesh Bala and Ngo Van Long, International Trade and Cultural Diversity: A Model of Preference Selection, July 2004

1243 Wolfgang Eggert and Alfons J. Weichenrieder, On the Economics of Bottle Deposits, July 2004

1244 Sören Blomquist and Vidar Christiansen, Taxation and Heterogeneous Preferences, July 2004

1245 Rafael Lalive and Alois Stutzer, Approval of Equal Rights and Gender Differences in Well-Being, July 2004

1246 Paolo M. Panteghini, Wide vs. Narrow Tax Bases under Optimal Investment Timing, July 2004

1247 Marika Karanassou, Hector Sala, and Dennis J. Snower, Unemployment in the European Union: Institutions, Prices, and Growth, July 2004

1248 Engin Dalgic and Ngo Van Long, Corrupt Local Government as Resource Farmers: The Helping Hand and the Grabbing Hand, July 2004

1249 Francesco Giavazzi and Guido Tabellini, Economic and Political Liberalizations, July 2004

1250 Yin-Wong Cheung and Jude Yuen, An Output Perspective on a Northeast Asia Currency Union, August 2004

1251 Ralf Elsas, Frank Heinemann, and Marcel Tyrell, Multiple but Asymmetric Bank Financing: The Case of Relationship Lending, August 2004

1252 Steinar Holden, Wage Formation under Low Inflation, August 2004

1253 Ngo Van Long and Gerhard Sorger, Insecure Property Rights and Growth: The Roles of Appropriation Costs, Wealth Effects, and Heterogeneity, August 2004 